\title{
Lattices acting on right-angled buildings
}

\author{
ANNE THOMAS
}

Let $X$ be a right-angled building. We show that the lattices in $\operatorname{Aut}(X)$ share many properties with tree lattices. For example, we characterise the set of covolumes of uniform and of nonuniform lattices in $\operatorname{Aut}(X)$, and show that the group $\operatorname{Aut}(X)$ admits an infinite ascending tower of uniform and of nonuniform lattices. These results are proved by constructing a functor from graphs of groups to complexes of groups.

$22 \mathrm{D} 05 ; 20 \mathrm{E} 42$

\section{Introduction}

Let $G$ be a locally compact topological group, with suitably normalised Haar measure $\mu$. A discrete subgroup $\Gamma \leq G$ is a lattice if the covolume $\mu(\Gamma \backslash G)$ is finite, and a uniform lattice if $\Gamma \backslash G$ is compact. A tower of lattices is a strictly increasing infinite chain of subgroups

$$
\Gamma_{1}<\Gamma_{2}<\cdots<\Gamma_{i}<\cdots
$$

such that each $\Gamma_{i}$ is a lattice in $G$. Two basic questions are:

(1) What are the possible covolumes of lattices in $G$ ?

(2) Does $G$ admit a tower of lattices?

These questions have been studied for many $G$. For example, if $G$ is a non-compact simple real Lie group, then the covolumes of lattices in $G$ are bounded away from 0 , and in most cases the set of lattice covolumes is discrete (see Lubotzky [15] and the references therein). A strong finiteness result for lattices in semisimple groups is that of Borel [4], which implies that for $G$ a higher-rank algebraic group over a local field, and any $c>0$, there are only finitely many lattices in $G$ with covolume less than $c$.

Nonclassical cases arise from the fact that if $G$ is the automorphism group of a locally finite polyhedral complex, then $G$ is naturally a locally compact group (see Section 1.1). Lattices in the automorphism groups of trees are treated by Bass and Lubotzky [3]. Lattices acting on a product of trees have been studied by, for example, Burger-Mozes [7]. 
We consider lattices in the automorphism groups of certain buildings. Let $P$ be a compact, convex polyhedron in $\mathbb{T}^{n}$ with all dihedral angles $\frac{\pi}{2}$, and let $(W, I)$ be the right-angled Coxeter system generated by reflections in the $(n-1)$-dimensional faces of $P$. A right-angled hyperbolic building of type $(W, I)$ is a polyhedral complex $X$, equipped with a maximal family of subcomplexes called apartments, each isometric to the tesselation of $\mathbb{M}^{n}$ by copies of $P$ (called chambers), satisfying the usual axioms for a building. A right-angled hyperbolic building has nonpositive curvature. It is locally the product of trees, but is not globally a product. In dimension 2, where right-angled hyperbolic buildings are sometimes known as "Bourdon buildings", $P$ is a regular right-angled hyperbolic polygon, and the link of each vertex of $X$ is a complete bipartite graph.

The dimension of a right-angled building with apartments isometric to $\mathbb{T}^{n}$ is at most 4, and this bound is sharp (see Potyagailo-Vinberg [16], and JanuszkiewiczŚwiątkowski [14]). However, given any right-angled Coxeter system $(W, I)$, there exist right-angled buildings with apartments isometric to the Davis complex for $(W, I)$; thus right-angled buildings may be constructed in arbitrarily high dimensions [14]. In Section 1.2 we give precise definitions and recall the classification of right-angled buildings. For work on hyperbolic buildings see, for example, Gaboriau-Paulin [10] and its references.

Our results for lattices acting on right-angled buildings are as follows.

Main Theorem Let $(W, I)$ be a right-angled Coxeter system and let $\left\{q_{i}\right\}_{i \in I}$ be a family of positive integers, $q_{i} \geq 2$, with $q_{i_{1}}=\max _{i}\left\{q_{i}\right\}$. Suppose $X$ is the (unique) building of type $(W, I)$, such that each $\{i\}$-residue of $X$ has cardinality $q_{i}$. Let $G=\operatorname{Aut}(X)$.

(1) Uniform covolumes

(a) If $m_{i_{1}, j}=\infty$ for some $j \in I$, then the set of covolumes of uniform lattices in $G$ is

$$
\mathcal{V}_{u}(G)=\left\{\frac{a}{b} \mid \text { prime divisors of } b \text { are strictly less than } q_{i_{1}}\right\}
$$

(b) If $m_{i_{1}, j}=\infty$ for some $j \in I$, and $q_{i_{1}}>2$, then for each $v \in \mathcal{V}_{u}(G)$ there is a countably infinite number of nonconjugate uniform lattices of covolume $v$.

\section{(2) Nonuniform covolumes}

(a) If there exist $i, j \in I$ such that $m_{i, j}=\infty$ and $q_{i}>2$, then the set of covolumes of nonuniform lattices is $(0, \infty)$.

(b) If there exist $i, j \in I$ such that $m_{i, j}=\infty$ and $q_{i}, q_{j}>2$, then for every $v>0$ there exist uncountably many commensurability classes of nonuniform lattices of covolume $v$. 
(3) Towers

(a) If there exist $i, j \in I$ such that $m_{i, j}=\infty$ and $q_{i}>2$, then there exists a tower of uniform lattices in $G$.

(b) If there exist $i, j \in I$ such that $m_{i, j}=\infty$ and $q_{i}>2$, then there exists a tower of nonuniform lattices in $G$.

(c) If there exist $i, j \in I$ such that $m_{i, j}=\infty$ and $q_{i}$ is composite, then there exists a tower of uniform lattices in $G$ such that the quotient by each lattice is isometric to a chamber of $X$.

We observe that if $(W, I)$ is generated by reflections in the faces of a right-angled hyperbolic polyhedron, then for each $i \in I$, there is a $j \in I$ so that $m_{i, j}=\infty$.

Although the right-angled building $X$ is a higher-dimensional object, the properties of lattices in $\operatorname{Aut}(X)$ listed in the Main Theorem are quite different to those of lattices in higher-rank algebraic groups. For example, the results cited above imply that semisimple groups do not admit lattices of arbitrarily small covolume, thus do not admit towers of (any) lattices. The theorem of Borel also implies that any $v>0$ is the covolume of at most finitely many lattices. We note too that there exist nonpositively curved polyhedral complexes whose automorphism groups, while nondiscrete, do not admit towers. For example the Bruhat-Tits building for $S L_{3}\left(\mathbb{Q}_{p}\right)$ has automorphism group containing $S L_{3}\left(\mathbb{Q}_{p}\right)$ as a finite index subgroup.

In contrast, by comparing the Main Theorem with results for trees, we see that the lattices in $G=\operatorname{Aut}(X)$ share many properties with tree lattices.

Uniform covolumes Rosenberg [17, Proposition 9.1.2] showed that the set of covolumes of uniform lattices acting on the $m$-regular tree is

$$
\left\{\frac{a}{b} \mid \text { prime divisors of } b \text { are strictly less than } m\right\}
$$

and found a similar result for biregular trees [17, Theorem 9.2.1].

Note that if all $q_{i}=2$ then the set of uniform covolumes is discrete. However, if some $q_{i}>2$ then the set of (uniform) covolumes is dense in $(0, \infty)$, and, in particular, $G$ admits (uniform) lattices of arbitrarily small covolume, as is the case for trees.

Our proof of the counting result (1)(b) will show that this property holds for regular and biregular trees as well (see Proposition 3.2 below). This generalises a result of Bass and Kulkarni [2, Theorem 7.1(b)] and answers a question put to us by A Lubotzky.

Nonuniform covolumes Bass-Lubotzky [3, Theorem 4.3] showed that for the $m-$ regular tree, $m \geq 3$, every $v>0$ is the covolume of some nonuniform lattice. Rosenberg [17, Theorem 8.2.2] extended this result to biregular trees. 
Farb-Hruska [9] constructed commensurability invariants for lattices acting on the $(m, n)$-biregular tree, for $m, n \geq 3$, and used these to show that for every $v>0$ there are uncountably many commensurability classes of nonuniform tree lattices [9, Corollary 1.2]. Farb-Hruska have shown (2)(a) and (2)(b) for some 2-dimensional right-angled hyperbolic buildings.

Towers Rosenberg showed [17, Theorem 3.3.1] that if $T$ is a tree such that $\operatorname{Aut}(T)$ is nondiscrete and admits a uniform lattice, then $\operatorname{Aut}(T)$ admits a tower of uniform lattices, and Carbone-Rosenberg showed [8, Theorem 5.4] that, with one exception, if $\operatorname{Aut}(T)$ admits a nonuniform lattice then it admits a tower of nonuniform lattices.

Part (3)(c) of the Main Theorem addresses the following finer question about towers. Let $X$ be a polyhedral complex and $G=\operatorname{Aut}(X)$. A subgroup of $G$ is homogeneous if it acts transitively on the cells of maximum dimension in $X$. Does $G$ admit a tower of homogeneous lattices?

When $X$ is the 3-regular tree, a deep theorem of Goldschmidt [12] implies that $G$ does not admit such a tower, since $G$ has only finitely many conjugacy classes of homogeneous lattices. The Goldschmidt-Sims conjecture (see Glasner [11]), which remains open, is that if $X$ is the $(p, q)$-biregular tree, where $p$ and $q$ are prime, then there are only finitely many conjugacy classes of homogeneous lattices in $G$. If $X$ is the product of two trees of prime valence, Glasner [11] has shown that there are only finitely many conjugacy classes of (irreducible) homogeneous lattices. We do not know if $G$ of the Main Theorem admits a homogeneous tower when all $q_{i}$ are prime. We note that, by the Functor Theorem below, if such a $G$ does have only finitely many conjugacy classes of homogeneous lattices, then the Goldschmidt-Sims conjecture holds.

The key fact used to prove the Main Theorem is that if a tree is "nicely embedded" in a right-angled building, then any group of automorphisms of the tree may be extended to a group of automorphisms of the building. We express this by means of a functor from graphs of groups to complexes of groups:

Functor Theorem Let $(W, I)$ and $X$ be as in the Main Theorem. For each $i, j \in I$ such that $m_{i, j}=\infty$, let $T$ be the $\left(q_{i}, q_{j}\right)$-biregular tree. Then there is a functor from the category of graphs of groups with universal covering tree $T$ to the category of complexes of groups with universal cover $X$, provided either that $X$ is "sufficiently symmetric", or that we restrict to 2-colourable graphs. Moreover, this functor takes coverings to coverings, and faithful graphs of groups to faithful complexes of groups.

We give a precise statement and proof of the Functor Theorem in Section 2.2, and then apply it to prove the Main Theorem in Section 3. An example of a sufficiently 
symmetric building $X$ is one with regular chambers, for instance if $\operatorname{dim}(X)=2$, and all $q_{i}$ equal. We note that there are many variants of the Functor Theorem, allowing promotion of known constructions of tree lattices to more general complexes of groups. For the theory of graphs of groups and their morphisms, see Section 1.3 and Serre [19], Bass [1] and Bass-Lubotzky [3]. The theory of complexes of groups (see Bridson and Haefliger [6]) is outlined in Section 1.4.

I thank Benson Farb for introducing me to this area, his enthusiasm and guidance, and his many comments on earlier versions of this paper. I would also like to thank G. Christopher Hruska and Frédéric Haglund for helpful discussions, and the anonymous referee for many worthwhile suggestions.

\section{Background}

Section 1.1 gives the basic definitions for lattices and describes a suitable normalisation of Haar measure for automorphism groups of polyhedral complexes. In Section 1.2 we discuss right-angled buildings. The key definitions for graphs of groups are given in Section 1.3, and those for complexes of groups in Section 1.4. We refer the reader to Bridson and Haefliger [6] for generalities on polyhedral complexes.

\subsection{Lattices and covolumes}

Let $G$ be a locally compact topological group with left-invariant Haar measure $\mu$. A discrete subgroup $\Gamma \leq G$ is a lattice if the covolume $\mu(\Gamma \backslash G)$ is finite. A lattice $\Gamma$ is uniform if $\Gamma \backslash G$ is compact. Let $S$ be a left $G$-set such that for every $s \in S$, the stabiliser $G_{S}$ is compact and open. Then if $\Gamma \leq G$ is discrete, the stabilisers $\Gamma_{s}$ are finite. We define the $S$-covolume of $\Gamma$ by

$$
\operatorname{Vol}(\Gamma \backslash \backslash S):=\sum_{s \in \Gamma \backslash S} \frac{1}{\left|\Gamma_{s}\right|} \leq \infty
$$

The following theorem shows that Haar measure may be normalised so that $\mu(\Gamma \backslash G)$ equals the $S$-covolume.

Theorem 1.1 (Serre [18]) Let $G$ be a locally compact topological group acting on a set $S$ with compact open stabilisers and a finite quotient $G \backslash S$. Suppose further that $G$ admits at least one lattice. Then there is a normalisation of the Haar measure $\mu$, depending only on the choice of $G$-set $S$, such that for each discrete subgroup $\Gamma$ of $G$ we have $\mu(\Gamma \backslash G)=\operatorname{Vol}(\Gamma \backslash \backslash S)$. 
Let $X$ be a connected, locally finite $n$-dimensional polyhedral complex, with $X_{n}$ the set of $n$-dimensional cells of $X$. Let $\operatorname{Aut}(X)$ be the group of cellular isometries of $X$. A subgroup of $\operatorname{Aut}(X)$ is said to act without inversions if its elements fix pointwise each cell that they preserve. The group $G=\operatorname{Aut}(X)$ is a locally compact topological group, with a neighbourhood basis of the identity consisting of automorphisms fixing larger and larger combinatorial balls. By the same arguments as for tree lattices (see Bass and Lubotzky [3, Chapter 1]), it can be shown that if $G \backslash X$ is finite, then a discrete subgroup $\Gamma \leq G$ is a lattice if and only if its $X_{n}$-covolume converges, and $\Gamma$ is uniform if and only if this sum has finitely many terms. Using Theorem 1.1, we now normalise the Haar measure $\mu$ on $G=\operatorname{Aut}(X)$ so that for all lattices $\Gamma \leq G$, the covolume of $\Gamma$ is

$$
\mu(\Gamma \backslash G)=\operatorname{Vol}\left(\Gamma \backslash \backslash X_{n}\right)
$$

\subsection{Right-angled buildings}

Let $(W, I)$ be a right-angled Coxeter system. Let $N$ be the finite nerve of $(W, I)$ and let $P^{\prime}$ be the simplicial cone on $N^{\prime}$ with vertex $x_{0}$. We write $\mathcal{S}^{f}$ for the set of $J \subseteq I$ such that the subgroup $W_{J}$ of $W$ generated by $J$ is finite. By convention, $W_{\phi}=1$, so the empty set $\phi$ is in $\mathcal{S}^{f}$. There is then a one-to-one correspondence between the vertices of $P^{\prime}$ and the types $J \in \mathcal{S}^{f}$. For each $i \in I$, the vertex of $P^{\prime}$ of type $\{i\}$ will be called an $i$-vertex, and the union of the simplices of $P^{\prime}$ which contain the $i$-vertex but not the cone point $x_{0}$ will be called an $i$-face.

A right-angled building of type $(W, I)$ is a polyhedral complex $X$ equipped with a maximal family of subcomplexes, called apartments. Each apartment is polyhedrally isometric to the Davis complex for $(W, I)$, and the copies of $P^{\prime}$ in $X$ are called chambers. The apartments and chambers of $X$ satisfy the usual axioms for a BruhatTits building. By abuse of notation, if $\operatorname{dim}(X)=n$ then we write $X_{n}$ for the set of chambers of $X$.

Each vertex of a right-angled building $X$ has a type $J \in \mathcal{S}^{f}$, induced by the types of $P^{\prime}$. For $i \in I$, an $\{i\}-$ residue of $X$ is then the connected subcomplex consisting of all chambers which meet a given $i$-face. Suppose $q_{i}$ is the cardinality of each $\{i\}$-residue of $X$, that is, the number of copies of $P^{\prime}$ in each $\{i\}$-residue. If $x$ is a vertex of $X$, of type some $J \in \mathcal{S}^{f}$ with $|J|=\operatorname{dim}(X)=n$, then the link of $x$ in $X$ is the join of $n$ sets of points of cardinalities respectively $q_{j}$, for $j \in J$.

If the Coxeter system $(W, I)$ is generated by reflections in the faces of an $n$-dimensional right-angled hyperbolic polyhedron $P$, then $P^{\prime}$ is the barycentric subdivision of $P$. The apartments of $X$ are isometric to $\mathbb{H}^{n}$, and we refer to $X$ as a hyperbolic building. For example, in Bourdon's building $I_{p, q}, P$ is a regular right-angled hyperbolic $p$-gon 
(see Bourdon [5]). The link of each vertex of $X$ is the complete bipartite graph $K_{q, q}$, which may be thought of as the join of 2 sets of $q$ points, and each $\{i\}$-residue consists of $q$ copies of $P$, glued together along a common edge.

The following result classifies right-angled buildings.

Theorem 1.2 (Haglund-Paulin [13, Proposition 1.2]) Let $(W, I)$ be a right-angled Coxeter system and $\left\{q_{i}\right\}_{i \in I}$ a family of positive integers $\left(q_{i} \geq 2\right)$. Then, up to isometry, there exists a unique building $X$ of type $(W, I)$, such that for each $i \in I$, the $\{i\}-$ residue of $X$ has cardinality $q_{i}$.

In the 2-dimensional case, this result is due to Bourdon [5]. According to Hagland and Paulin [13], Theorem 1.2 was proved by M Globus, and was known also to M Davis, T Januszkiewicz and J Świątkowski.

\subsection{Graphs of groups}

We give only the definitions most relevant to the proof of the Functor Theorem below. See Serre [19], Bass-Lubotzky [3] and Bass [1] for more complete treatments.

A graph of groups $\mathbb{A}=(A, \mathcal{A})$ consists of a connected graph $A$, with vertices $V(A)$ and edges $E(A)$, together with groups $\mathcal{A}_{v}$ for each $v \in V(A)$ and $\mathcal{A}_{e}=\mathcal{A}_{\bar{e}}$ for each $e \in E(A)$, and monomorphisms $\alpha_{e}: \mathcal{A}_{e} \rightarrow \mathcal{A}_{i(e)}$. See Bass [1] for the definitions of the path group $\pi(\mathbb{A})$, the fundamental group of the graph of groups $\pi_{1}\left(\mathbb{A}, v_{0}\right)$ and the universal covering tree. A graph of groups is faithful if its fundamental group acts faithfully on its universal covering tree.

Let $\mathbb{A}=(A, \mathcal{A})$ and $\mathbb{B}=(B, \mathcal{B})$ be graphs of groups. A morphism $\phi: \mathbb{A} \rightarrow \mathbb{B}$ consists of:

(1) a morphism of graphs $f: A \rightarrow B$;

(2) homomorphisms of local groups $\phi_{v}: \mathcal{A}_{v} \rightarrow \mathcal{B}_{f(v)}$ and $\phi_{e}=\phi_{\bar{e}}: \mathcal{A}_{e} \rightarrow \mathcal{B}_{f(e)}$; and

(3) elements $\gamma_{v} \in \pi_{1}(\mathbb{B}, f(v))$ for each $v \in V(A)$, and $\gamma_{e} \in \pi(\mathbb{B})$ for each $e \in E(A)$, such that if $v=i(e)$ then

(a) $\delta_{e}:=\gamma_{v}^{-1} \gamma_{e} \in \mathcal{B}_{f(v)}$; and

(b) $\phi_{a} \circ \alpha_{e}=\operatorname{Ad}\left(\delta_{e}\right) \circ \alpha_{f(e)} \circ \phi_{e}$, where $\operatorname{Ad}\left(\delta_{e}\right)$ is conjugation by $\delta_{e}$ in $\mathcal{B}_{f(v)}$.

The morphism $\phi$ is a covering if in addition:

(1) each $\phi_{v}$ and $\phi_{e}$ is injective; and 
(2) for each $v \in V(A)$ and $e^{\prime} \in E(B)$ with $i\left(e^{\prime}\right)=f(v)$, the map

$$
\coprod_{\substack{e \in f^{-1}\left(e^{\prime}\right) \\ i(e)=v}} \mathcal{A}_{v} / \alpha_{e}\left(\mathcal{A}_{e}\right) \rightarrow \mathcal{B}_{f(v)} / \alpha_{e^{\prime}}\left(\mathcal{B}_{e^{\prime}}\right)
$$

induced by $g \mapsto \phi_{v}(g) \delta_{e}$ is a bijection.

\subsection{Complexes of groups}

We sketch the theory of complexes of groups, due to Haefliger [6]. Section 1.4.1 outlines the construction to be used for towers of lattices.

Throughout this section, if $X$ is a polyhedral complex, then $X^{\prime}$ is the first barycentric subdivision of $X$. This is a simplicial complex with vertices $V\left(X^{\prime}\right)$ and edges $E\left(X^{\prime}\right)$. Each $a \in E\left(X^{\prime}\right)$ corresponds to cells $\tau \subset \sigma$ of $X$, and so may be oriented from $\sigma$ to $\tau$. We write $i(a)=\sigma$ and $t(a)=\tau$. Two edges $a$ and $b$ of $X^{\prime}$ are composable if $i(a)=t(b)$, in which case there exists an edge $c=a b$ of $X^{\prime}$ such that $i(c)=i(b)$, $t(c)=t(a)$ and $a, b$ and $c$ form the boundary of a 2-simplex in $X^{\prime}$.

A complex of groups $G(X)=\left(G_{\sigma}, \psi_{a}, g_{a, b}\right)$ over a polyhedral complex $X$ is given by:

(1) a group $G_{\sigma}$ for each $\sigma \in V\left(X^{\prime}\right)$, called the local group at $\sigma$;

(2) a monomorphism $\psi_{a}: G_{i(a)} \rightarrow G_{t(a)}$ for each $a \in E\left(X^{\prime}\right)$; and

(3) for each pair of composable edges $a, b$ in $X^{\prime}$, an element $g_{a, b} \in G_{t(a)}$, such that

$$
\operatorname{Ad}\left(g_{a, b}\right) \circ \psi_{a b}=\psi_{a} \circ \psi_{b}
$$

where $\operatorname{Ad}\left(g_{a, b}\right)$ is conjugation by $g_{a, b}$ in $G_{t(a)}$, and for each triple of composable edges $a, b, c$ the following cocycle condition holds

$$
\psi_{a}\left(g_{b, c}\right) g_{a, b c}=g_{a, b} g_{a b, c}
$$

All complexes of groups in this paper will be simple, meaning that each $g_{a, b}$ is trivial.

Next we define morphisms of complexes of groups. Let $G(X)=\left(G_{\sigma}, \psi_{a}\right)$ and $H(Y)=\left(H_{\tau}, \psi_{b}\right)$ be simple complexes of groups over polyhedral complexes $X$ and $Y$. Let $f: X^{\prime} \rightarrow Y^{\prime}$ be a simplicial map sending vertices to vertices and edges to edges (such an $f$ is nondegenerate). A morphism $\phi: G(X) \rightarrow H(Y)$ over $f$ consists of:

(1) a homomorphism $\phi_{\sigma}: G_{\sigma} \rightarrow H_{f(\sigma)}$ for each $\sigma \in V\left(X^{\prime}\right)$; and 
(2) an element $\phi(a) \in H_{t(f(a))}$ for each $a \in E\left(X^{\prime}\right)$, such that

$$
\operatorname{Ad}(\phi(a)) \circ \psi_{f(a)} \circ \phi_{i(a)}=\phi_{t(a)} \circ \psi_{a}
$$

and for all pairs of composable edges $(a, b)$ in $E\left(X^{\prime}\right)$,

$$
\phi(a b)=\phi(a) \psi(\phi(b))
$$

We note that morphisms may also be defined over degenerate maps $f: X^{\prime} \rightarrow Y^{\prime}$. If $f$ is an isometry and each $\phi_{\sigma}$ an isomorphism then $\phi$ is an isomorphism. A morphism $\phi: G(X) \rightarrow H(Y)$ is a covering if:

(1) each $\phi_{\sigma}$ is injective; and

(2) for each $\sigma \in V\left(X^{\prime}\right)$ and $b \in E\left(Y^{\prime}\right)$ such that $t(b)=f(\sigma)$, the map

$$
\coprod_{\substack{a \in f^{-1}(b) \\ t(a)=\sigma}} G_{\sigma} / \psi_{a}\left(G_{i(a)}\right) \rightarrow H_{f(\sigma)} / \psi_{b}\left(H_{i(b)}\right)
$$

induced by $g \mapsto \phi_{\sigma}(g) \phi(a)$ is a bijection.

Let $G$ be a group acting without inversions on a polyhedral complex $Y$. This action induces a complex of groups, as follows. Let $X=G \backslash Y$ with $p: Y \rightarrow X$ the natural projection. For each $\sigma \in V\left(X^{\prime}\right)$, choose $\tilde{\sigma} \in V\left(Y^{\prime}\right)$ such that $p(\widetilde{\sigma})=\sigma$. The local group $G_{\sigma}$ is the stabiliser of $\tilde{\sigma}$ in $G$, and the $\psi_{a}$ and $g_{a, b}$ are defined using further choices. The resulting complex of groups $G(X)$ is unique up to isomorphism. A complex of groups is developable if it is isomorphic to a complex of groups associated, as just described, to an action.

Let $G(X)$ be a (simple) complex of groups, and choose a basepoint $\sigma_{0} \in V\left(X^{\prime}\right)$. The fundamental group of the complex of groups $\pi_{1}\left(G(X), \sigma_{0}\right)$ is defined so that if $X$ is simply connected, $\pi_{1}\left(G(X), \sigma_{0}\right)$ is isomorphic to the direct limit of the family of groups $G_{\sigma}$ and monomorphisms $\psi_{a}$.

If $G(X)$ is developable, then it has a universal cover $\widetilde{G(X)}$. This is a simply-connected polyhedral complex, equipped with an action of $\pi_{1}\left(G(X), \sigma_{0}\right)$, so that the complex of groups induced by the action of the fundamental group on the universal cover is isomorphic to $G(X)$.

We now describe a geometric condition for developability. Let $X$ be a connected polyhedral complex and $\sigma \in V\left(X^{\prime}\right)$. The star of $\sigma$, written $\operatorname{St}(\sigma)$, is the union of the interiors of the simplices in $X^{\prime}$ which meet $\sigma$. If $G(X)$ is a complex of groups over $X$ then, even if $G(X)$ is not developable, each $\sigma \in V\left(X^{\prime}\right)$ has a local development. That is, we may associate to $\sigma$ an action of $G_{\sigma}$ on the $\operatorname{star} \operatorname{St}(\widetilde{\sigma})$ of a vertex $\widetilde{\sigma}$ in 
some simplicial complex, such that $\operatorname{St}(\sigma)$ is the quotient of $\operatorname{St}(\widetilde{\sigma})$ by the action of $G_{\sigma}$. If $G(X)$ is developable, then for each $\sigma \in V\left(X^{\prime}\right)$, the local development of $\sigma$ is isomorphic to the star of each lift $\widetilde{\sigma}$ of $\sigma$ in the universal cover $\widetilde{G(X)}$.

The local development $\operatorname{St}(\widetilde{\sigma})$ has a metric structure induced by that of the polyhedral complex $X$. We say that a complex of groups $G(X)$ is nonpositively curved if for all $\sigma \in V\left(X^{\prime}\right), \operatorname{St}(\widetilde{\sigma})$ has nonpositive curvature (that is, $\operatorname{St}(\widetilde{\sigma})$ is locally $\operatorname{CAT}(\kappa)$ for some $\kappa \leq 0)$ in this induced metric. The importance of this condition is given by:

Theorem 1.3 (Haefliger [6]) A nonpositively curved complex of groups is developable.

Let $G(X)$ be a developable complex of groups over a polyhedral complex $X$, with universal cover $Y$ and fundamental group $\Gamma$. We say that $G(X)$ is faithful if the action of $\Gamma$ on $Y$ is faithful. If $G(X)$ is faithful, then $\Gamma$ may be regarded as a subgroup of $\operatorname{Aut}(Y)$. Moreover, $\Gamma$ is discrete if and only if all local groups of $G(X)$ are finite, and a discrete $\Gamma$ is a uniform lattice if and only if $X$ is a finite polyhedral complex.

1.4.1 Towers Let $G(X)=\left(G_{\sigma}, \psi_{a}\right)$ and $H(X)=\left(H_{\tau}, \psi_{b}\right)$ be simple complexes of groups over a complete, connected polyhedral complex $X$. We say that $G(X)$ is a full complex of subgroups of $H(X)$ if there is a covering $\phi: G(X) \rightarrow H(X)$ over the identity map $X^{\prime} \rightarrow X^{\prime}$ such that each $\phi(a)=1$. By [6, Corollary 3.16, Chapter III. $\left.\mathcal{G}\right]$ the covering $\phi$ induces an injective homomorphism of fundamental groups

$$
\pi_{1}\left(G(X), \sigma_{0}\right) \rightarrow \pi_{1}\left(H(X), \sigma_{0}\right)
$$

Suppose now that $Y$ is a polyhedral complex, and that we have a sequence $\left(G_{i}(X)\right)$ of complexes of groups over $X$, such that

(1) for each $i, G_{i}(X)$ is a full complex of subgroups of $G_{i+1}(X)$ with respect to a covering $\phi_{i}$;

(2) the image of each $\left(\phi_{i}\right)_{\sigma}$ is a proper subgroup; and

(3) $Y$ is the universal cover of each $G_{i}(X)$.

The sequence $G_{i}(X)$ then induces an infinite strictly ascending chain of fundamental groups

$$
\pi_{1}\left(G_{1}(X), \sigma_{0}\right)<\pi_{1}\left(G_{2}(X), \sigma_{0}\right)<\cdots<\pi_{1}\left(G_{i}(X), \sigma_{0}\right)<\cdots
$$

If each $G_{i}(X)$ is a faithful complex of finite groups, with finite covolume, then each fundamental group in this chain is a lattice in $\operatorname{Aut}(Y)$. In this way we may construct towers of lattices in $\operatorname{Aut}(Y)$. 


\section{The Functor Theorem}

This section contains our key technical result, the Functor Theorem below. Let $\mathcal{G}$ be the category of graphs of groups and morphisms, as defined by Bass [1]. Let $\mathcal{C}$ be the category of complexes of groups and morphisms, as defined by Haefliger [6]. In Section 2.1 we make precise the relationship between $\mathcal{G}$ and the subcategory of $\mathcal{C}$ consisting of complexes of groups over 1-dimensional polyhedral complexes. This is used in Section 2.2 where we state and prove the Functor Theorem.

\subsection{Relationship between graphs of groups and 1-dimensional complexes of groups}

Complexes of groups are generalisations of graphs of groups. However, Haefliger remarks [6, page 566] that it is unclear whether the notion of morphism is the same. Let $\mathcal{C}_{1}$ be the subcategory of $\mathcal{C}$ consisting of complexes of groups over 1-dimensional polyhedral complexes (that is, simplicial graphs), and morphisms over nondegenerate polyhedral maps. The following result specifies the relationship between the category $\mathcal{G}$ of graphs of groups and the category $\mathcal{C}_{1}$.

Proposition 2.1 There is a functor $F: \mathcal{G} \rightarrow \mathcal{C}_{1}$ which is a bijection on objects, takes faithful graphs of groups to faithful complexes of groups, surjects onto the set of morphisms of $\mathcal{C}_{1}$ and takes coverings to coverings. However, there is no functor from $\mathcal{G}$ to $\mathcal{C}_{1}$ which is injective on the set of morphisms of $\mathcal{G}$. Hence these categories are not isomorphic.

Proof We first define the functor $F$ on objects. Let $\mathbb{A}=(A, \mathcal{A})$ be a graph of groups and let $|A|$ be the geometric realisation of the graph $A$. We construct a complex of groups $F(\mathbb{A})$ over $|A|$. The local groups at the vertices of $|A|$ are the vertex groups of $\mathbb{A}$. For each $e \in E(A)$, let $\sigma_{e}=\sigma_{\bar{e}}$ be the vertex of the barycentric subdivision $|A|^{\prime}$ at the midpoint of $e$. Then the local group at $\sigma_{e}$ in the complex of groups is $\mathcal{A}_{e}=\mathcal{A}_{\bar{e}}$. Each monomorphism $\alpha_{e}: \mathcal{A}_{e} \rightarrow \mathcal{A}_{i(e)}$ of the graph of groups induces the same monomorphism of corresponding local groups in the complex of groups $F(\mathbb{A})$.

It is clear that $F$ is a bijection from the set of objects of $\mathcal{G}$ to the set of objects of $\mathcal{C}_{1}$. From the definitions of the actions on the respective universal covers, one sees also that faithful graphs of groups are mapped to faithful complexes of groups.

To define the functor $F$ on morphisms, let $\phi: \mathbb{A} \rightarrow \mathbb{B}$ be a morphism of graphs of groups over a graph morphism $f: A \rightarrow B$. By abuse of notation $f$ induces a nondegenerate polyhedral map $f:|A|^{\prime} \rightarrow|B|^{\prime}$. We define a morphism of complexes 
of groups $F(\phi): F(\mathbb{A}) \rightarrow F(\mathbb{B})$ over $f$. The homomorphisms on local groups are the same as for the morphism $\phi$. Let $a$ be an edge of $|A|^{\prime}$. Then the monomorphism $\psi_{a}$ in the complex of groups $F(\mathbb{A})$ is the same as some $\alpha_{e}: \mathcal{A}_{e} \rightarrow \mathcal{A}_{i(e)}$ in the graph of groups $\mathbb{A}$. Put $\phi(a)=\delta_{e}$, and we obtain the required

$$
\phi_{t(a)} \circ \psi_{a}=\operatorname{Ad}(\phi(a)) \circ \psi_{f(a)} \circ \phi_{i(a)}
$$

Hence $F(\phi)$ is a morphism of complexes of groups. The map $F$ respects composition of morphisms, and is thus a functor.

Given a morphism of $\mathcal{C}_{1}$, one can construct a morphism of the corresponding objects in $\mathcal{G}$ by setting all $\gamma_{v}=1$, and all $\gamma_{e}=\phi(a)$, where the monomorphism $\alpha_{e}$ is the same as the monomorphism $\psi_{a}$. Hence $F$ surjects onto the set of morphisms of $\mathcal{C}_{1}$. By checking the definition of covering in both categories, we find that $F$ sends coverings to coverings, and surjects onto the set of coverings of $\mathcal{C}_{1}$.

However, since many choices of $\gamma_{v}$ and $\gamma_{e}$ could lead to the same collection of $\delta_{e}$, there is no functor from $\mathcal{G}$ to $\mathcal{C}_{1}$ which is injective on the set of morphisms of $\mathcal{G}$.

\subsection{The Functor Theorem}

Let $T$ be a tree and $X$ a right-angled building. We write $\mathcal{G}(T)$ for the category of graphs of groups with universal covering tree $T$ and $\mathcal{C}(X)$ for the category of developable complexes of groups with universal cover $X$. We now construct a functor from $\mathcal{G}(T)$ to $\mathcal{C}(X)$, for certain $T$ and $X$.

This functor will exist only when the building $X$ is "sufficiently symmetric", as defined below. However, the proof of the Functor Theorem will show that even if $X$ is not sufficiently symmetric, we can construct a functor taking faithful graphs of groups to faithful complexes of groups, and coverings to coverings, if we restrict to graphs of groups whose vertices are 2-colourable and to coverings which preserve this colouring. As shown in Section 3 below, the proof of the Main Theorem requires only this restricted functor.

To explain when a right-angled building $X$ is sufficiently symmetric, let $(W, I)$ be the right-angled Coxeter system, $P^{\prime}$ the chamber and $\left\{q_{i}\right\}$ the parameters associated to $X$. Suppose $m_{i_{1}, i_{2}}=\infty$, and consider the following symmetry conditions:

(1) there exists a bijection $g$ on the set $I$ such that $m_{i, j}=m_{g(i), g(j)}$ for all $i, j \in I$, and $g\left(i_{1}\right)=i_{2}$. This may be thought of as an isometry of $P^{\prime}$ which takes the $i_{1}$-face to the $i_{2}$-face. 
(2) there exists a bijection

$$
h:\left\{i \in I \mid m_{i_{1}, i}<\infty\right\} \rightarrow\left\{i \in I \mid m_{i_{2}, i}<\infty\right\}
$$

such that $m_{i, j}=m_{h(i), h(j)}$ for all $i, j$ in the domain, $h\left(i_{1}\right)=i_{2}$, and $q_{i}=q_{h(i)}$ for all $i$ in the domain. This may be thought of as an isometry from the simplicial neighbourhood in $P^{\prime}$ of the $i_{1}$-face to the simplicial neighbourhood in $P^{\prime}$ of the $i_{2}$-face, which preserves the cardinalities $q_{i}$.

Conditions (1) and (2) are satisfied if, for example, $P^{\prime}$ is the barycentric subdivision of a regular right-angled hyperbolic polyhedron $P$, and all $q_{i}$ are equal. In dimension 2, $P$ may be a $k$-gon for any $k \geq 5$. In each of dimensions 3 and 4 , there is only one such $P$, the dodecahedron and the 120-cell respectively (see, for example, Vinberg and Shvartsman [21]).

Conditions (1) and (2) may also be satisfied for $P$ which is not regular. For example, suppose $P$ is 3 -dimensional. We claim that there exist $i_{1}, i_{2} \in I$ such that $m_{i_{1}, i_{2}}=\infty$ and the $i_{1}$ - and $i_{2}$-faces of $P$ are isometric. Since the 2-dimensional faces of $P$ are regular, it suffices to exhibit a pair of nonadjacent 2-dimensional faces with, say, 5 sides. Let $a_{2}$ be the number of 2-dimensional faces of $P$. For each 2-dimensional face $F$, let $a_{1}(F)$ be the number of sides of $F$ and let $\operatorname{ex}(F)=a_{1}(F)-5 \geq 0$. Elementary calculations yield

$$
a_{2}=12+\sum_{\operatorname{dim}(F)=2} \operatorname{ex}(F)
$$

(see Potyagailo and Vinberg [16, page 70]). This implies that there are at least 12 faces $F$ with 5 sides. Hence we can find two nonadjacent 2-dimensional faces of $P$ with 5 sides. This kind of argument cannot be extended to dimension 4 , since the 3-dimensional faces of $P$ will not in general be regular.

We now state and prove the Functor Theorem.

Functor Theorem Let $X$ be a right-angled building of type $(W, I)$ and parameters $\left\{q_{i}\right\}$. For each $i_{1}, i_{2} \in I$ such that $m_{i_{1}, i_{2}}=\infty$, let $T$ be the $\left(q_{i_{1}}, q_{i_{2}}\right)$-biregular tree. Suppose condition (1) above holds, and that if $q_{i_{1}}=q_{i_{2}}$ then condition (2) above holds, with $g$ an extension of $h$. Then there is a functor $F$ from $\mathcal{G}(T)$ to $\mathcal{C}(X)$, which takes faithful graphs of groups to faithful complexes of groups, and coverings to coverings.

Proof By Proposition 2.1, it suffices to construct a functor $F$ from the image of $\mathcal{G}(T)$ in $\mathcal{C}_{1}$ to $\mathcal{C}(X)$. We first define $F$ on objects. Let $G(Y)$ be an object of $\mathcal{C}_{1}$ which is in the image of $\mathcal{G}(T)$.

Algebraic 83 Geometric Topology, Volume 6 (2006) 
For each edge $e$ of the simplicial graph $Y$, let $P_{e}^{\prime}$ be a copy of $P^{\prime}$ (recall that $P^{\prime}$ is the simplicial cone with vertex $x_{0}$ on the barycentric subdivision of the finite nerve of $(W, I))$. Identify the midpoint of $e$ with the vertex $x_{0}$ of $P_{e}^{\prime}$.

Suppose first that the vertices of the graph $Y$ may be 2-coloured with the types $i_{1}$ and $i_{2}$. If $q_{i_{1}}=q_{i_{2}}$ choose such a 2-colouring, and if $q_{i_{1}} \neq q_{i_{2}}$, then assign the colours $i_{1}$ and $i_{2}$ according to the valences of the vertices in the universal covering tree $T$. Then for $j=1,2$ identify the vertex of $e$ of type $i_{j}$ with the $i_{j}$-vertex of $P_{e}^{\prime}$.

Now suppose the vertices of the graph $Y$ are not 2-colourable. If the edge $e$ does not form a loop in $Y$, then identify one vertex of $e$ with the $i_{1}$-vertex of $P_{e}^{\prime}$, and the other vertex with the $i_{2}$-vertex. If the edge $e$ does form a loop in $Y$ then use the isometry $h$ of condition (2) above to form $P_{e}^{\prime} / h$. That is, glue together the $i_{1}-$ and $i_{2}$-faces of $P_{e}^{\prime}$ using $h$. Then identify the vertex of $Y$ to which both ends of $e$ are joined with the image of the $i_{1}$ - and $i_{2}$-vertices in $P_{e}^{\prime} / h$.

Now, glue together, either by preserving type on $i_{1}-$ and $i_{2}$-faces, or if the vertices of $Y$ are not 2-colourable, by using the isometry $h$, the faces of the various $P_{e}^{\prime}$ and $P_{e}^{\prime} / h$ whose centres correspond to the same vertex of $Y$. Let $F(Y)$ be the resulting polyhedral complex. By construction, the barycentric subdivision $Y^{\prime}$ embeds in $F(Y)$. Note also that each vertex of $F(Y)$ has either the type of a unique $J \in \mathcal{S}^{f}$, or the two types $J$ and $h(J)$, where $i_{1} \in J$ and $J \in \mathcal{S}^{f}$.

We now construct a complex of groups $F(G(Y))$ over $F(Y)$. First, fix the local groups and monomorphisms induced by the embedding of $Y^{\prime}$ in $F(Y)$. For each $i \in I$, let $G_{i}=\mathbb{Z} / q_{i} \mathbb{Z}$. For each edge $e$ of $Y$, let $G_{e}$ be the local group at the midpoint of $e$ in the complex of groups $G(Y)$.

Consider $J \in \mathcal{S}^{f}$ which does not contain $i_{1}$ or $i_{2}$. The local group at the vertex with type $J$ of $P_{e}^{\prime}$ or $P_{e}^{\prime} / h$ is then

$$
G_{e} \times \prod_{j \in J} G_{j}
$$

The monomorphisms between such local groups are natural inclusions.

Now consider $J \in \mathcal{S}^{f}$ which contains one of $i_{1}$ or $i_{2}$ (since $m_{i_{1}, i_{2}}=\infty, J$ cannot contain both $i_{1}$ and $i_{2}$ ). Without loss of generality suppose $i_{1} \in J$, and let $F_{e}$ be either the $i_{1}$-face of $P_{e}^{\prime}$, or the glued face of $P_{e}^{\prime} / h$. The vertex of type $J$ in $P_{e}^{\prime}$ or $P_{e}^{\prime} / h$ is then contained in $F_{e}$. Let $v$ be the vertex of $Y$ which is identified with the centre of $F_{e}$, and let $G_{v}$ be the local group at $v$ in the complex of groups $G(Y)$. The local group at the vertex of type $J$ of $P_{e}^{\prime}$ or $P_{e}^{\prime} / h$ is then

$$
G_{v} \times \prod_{\substack{j \in J \\ j \neq i_{1}}} G_{j}
$$

Algebraic 83 Geometric Topology, Volume 6 (2006) 
This is well-defined, since if the face of type $J$ also has type $h(J)$, by condition (2) we have $q_{j}=q_{h(j)}$ for each $j \in J$, hence $G_{j}=G_{h(j)}=\mathbb{Z} / q_{j} \mathbb{Z}$. The monomorphism from $G_{v}$ to this local group is the natural inclusion onto the first factor. For each $J^{\prime} \subset J$ with $i_{1} \in J^{\prime}$, the monomorphism

$$
G_{v} \times \prod_{\substack{j \in J^{\prime} \\ j \neq i_{1}}} G_{j} \longrightarrow G_{v} \times \prod_{\substack{j \in J \\ j \neq i_{1}}} G_{j}
$$

is the natural inclusion. For each $J^{\prime} \subset J$ with $i_{1} \notin J^{\prime}$, the monomorphism

$$
G_{e} \times \prod_{j \in J^{\prime}} G_{j} \longrightarrow G_{v} \times \prod_{\substack{j \in J \\ j \neq i_{1}}} G_{j}
$$

is a monomorphism $G_{e} \rightarrow G_{v}$ from the complex of groups $G(Y)$ on the first factor, and natural inclusion on the other factors.

We now show that the complex of groups $F(G(Y))$ is developable, with universal cover $X$. Let $\tau$ be a vertex of $F(Y)$ of type $J$, with $|J|=n=\operatorname{dim}(X)$. One verifies that the link in the local development at $\tau$ is the join of $n$ sets of points of cardinalities respectively $q_{j}$, for $j \in J$. Thus, as $P^{\prime}$ is right-angled, by Gromov's Link Condition (see Bridson and Haefliger [6, Theorem 5.2, Chapter II]) each local development has nonpositive curvature. It follows by Theorem 1.3 above that the complex of groups $F(G(Y))$ is developable. The universal cover is a building (see Gaboriau and Paulin [10, Section 3.3]), and by Theorem 1.2 above, the universal cover is the unique right-angled building $X$ of type $(W, I)$ and parameters $\left\{q_{i}\right\}$.

The universal covering tree $T$ naturally embeds in $X$, and by construction, if the fundamental group of $G(Y)$ acts faithfully on $T$, then the fundamental group of $F(G(Y))$ acts faithfully on $T$, and hence on $X$. Thus $F$ maps faithful graphs of groups to faithful complexes of groups.

We now define the functor $F$ on morphisms. Let $G(Y)$ and $H(Z)$ be objects of $\mathcal{C}_{1}$ in the image of $\mathcal{G}(T)$, and let $\phi: G(Y) \rightarrow H(Z)$ be a morphism over a nondegenerate map $f: Y^{\prime} \rightarrow Z^{\prime}$. Let $F(Y)$ and $F(Z)$ be the polyhedral complexes constructed from the simplicial graphs $Y$ and $Z$ as above.

If the vertices of $Y$ and $Z$ are 2-colourable by the types $i_{1}$ and $i_{2}$, and $f$ preserves these types, then $f$ may be extended to a polyhedral map $F(f): F(Y) \rightarrow F(Z)$ by preserving type on each copy of $P^{\prime}$. Otherwise, we use condition (1) to construct $F(f): F(Y) \rightarrow F(Z)$. On each copy of $P^{\prime}$ or $P^{\prime} / h, F(f)$ maps the vertex of type $J$ to the vertex of type $g(J)$. This is well-defined since $h$ is the restriction of $g$. 
We now construct a morphism $F(\phi)$ of complexes of groups over $F(f)$. If $\tau$ is a vertex of $F(Y)$ then the local group at $\tau$ is a direct product

$$
G_{\sigma} \times \prod G_{j}
$$

where $\sigma$ is a vertex of $Y^{\prime}$. The homomorphism of local groups

$$
G_{\sigma} \times \prod G_{j} \longrightarrow H_{f(\sigma)} \times \prod G_{j}
$$

is $\phi_{\sigma}$ on the first factor, and the identity on other factors. Let $b$ be an edge of $F(Y)$. If $\psi_{b}$, the monomorphism along the edge $b$ in the complex of groups $F(G(Y))$, has as its first factor a monomorphism $\psi_{a}$ from the complex of groups $G(Y)$, put $F(\phi)(b)=\phi(a)$. Otherwise, put $F(\phi)(b)=1$.

The following claims all follow from definitions: $F(\phi)$ is a morphism of complexes of groups, $F(\phi)$ respects composition, and $F(\phi)$ takes coverings to coverings. This completes the proof of the Functor Theorem.

\section{Proof of the Main Theorem}

We conclude by proving the Main Theorem, stated in the Introduction. Most parts of the theorem are proved using the Functor Theorem. Throughout this section, $X$ is as in the statement of the Main Theorem, and $G=\operatorname{Aut}(X)$.

For covolume results, we will use Corollary 3.1 below, which follows from the proof of the Functor Theorem. Recall that for a polyhedral complex $Y$, we write $Y_{n}$ for the set of $n$-dimensional cells of $Y$.

Corollary 3.1 Suppose $G(Y)$ is a faithful complex of finite groups over an $n-$ dimensional polyhedral complex $Y$, with universal cover $X$. Let $\Gamma$ be the fundamental group of the complex of groups $G(Y)$. For each cell $\sigma \in Y_{n}$, let $\Gamma_{\sigma}$ be the local group at the barycentre of that cell. Then if $G(Y)=F(\mathbb{A})$, where $F$ is the functor defined in the Functor Theorem, and $\mathbb{A}=(A, \mathcal{A})$ is a graph of groups,

$$
\mu(\Gamma \backslash G)=\sum_{\sigma \in Y_{n}} \frac{1}{\left|\Gamma_{\sigma}\right|}=\sum_{e \in E(A)} \frac{1}{\left|\mathcal{A}_{e}\right|}
$$

That is, $\mu(\Gamma \backslash G)$ equals the covolume of the graph of groups $\mathbb{A}$. 


\subsection{Uniform covolumes}

3.1.1 Proof of (1)(a) We first show that the covolume of every uniform lattice belongs to the set $\mathcal{V}_{u}(G)$ of rationals given in (1)(a). Then we show that any $v \in \mathcal{V}_{u}(G)$ is the covolume of some uniform lattice.

Since $X$ is a building, the group $G=\operatorname{Aut}(X)$ has a finite index normal subgroup $\operatorname{Aut}_{0}(X)$, the group of type-preserving automorphisms, which acts without inversions. Thus, any uniform lattice $\Gamma<G$ has a finite index subgroup $\Gamma \cap \operatorname{Aut}_{0}(X)$ which acts without inversions. Now, the set $\mathcal{V}_{u}(G)$ is closed under multiplication by positive integers. Hence, to show that the covolume of every uniform lattice belongs to $\mathcal{V}_{u}(G)$, it suffices to consider only uniform lattices which act without inversions.

Let $\Gamma$ be a uniform lattice in $G$ which acts without inversions, and let

$$
\mu(\Gamma \backslash G)=\operatorname{Vol}\left(\Gamma \backslash \backslash X_{n}\right)=\frac{a}{b}
$$

To show that the prime divisors of $b$ are strictly less than $\max _{i}\left\{q_{i}\right\}$, we use the following. Let $x$ be a vertex of $X$, of type some $J \in \mathcal{S}^{f}$ with $|J|=n=\operatorname{dim}(X)$. Let $S_{k}$ be the symmetric group on $k$ letters. The group of type-preserving automorphisms of the link of $x$ in $X$ is then

$$
\prod_{j \in J} S_{q_{j}}
$$

and its subgroup which fixes an $(n-1)$-dimensional cell of the link pointwise is

$$
\operatorname{Fix}(n-1, J):=\prod_{j \in J} S_{q_{j}-1} .
$$

A result in a forthcoming paper [20, Theorem 3] then implies the restriction on the prime divisors of $b$; we sketch the argument. It suffices to show that for any $\sigma \in X_{n}$, the prime divisors of $\left|\Gamma_{\sigma}\right|$ are strictly less than $\max _{i}\left\{q_{i}\right\}$. Since $\Gamma_{\sigma}$ is a finite group, for some $m>0$ it injects into $H_{m}:=\operatorname{Aut}(B(m, \sigma))$, the group of automorphisms of the combinatorial ball of radius $m$ in $X$ centred at $\sigma$. It now suffices to bound the prime divisors of $\left|H_{m}\right|$. For this, we use induction on $m$, and the fact that the prime divisors of the group $\operatorname{Fix}(n-1, J)$ above are strictly less than $\max _{i}\left\{q_{i}\right\}$. We conclude that the covolume of any uniform lattice in $G$ belongs to the set $\mathcal{V}_{u}(G)$.

We now show that every $v \in \mathcal{V}_{u}(G)$ is the covolume of some uniform lattice. By Corollary 3.1 above, and the proof of the Functor Theorem, it suffices to show that, for any rational $\frac{a}{b}$ with the prime divisors of $b$ strictly less than $\max _{i}\left\{q_{i}\right\}$, there is a faithful graph of finite groups $\mathbb{A}=(A, \mathcal{A})$ over a finite graph $A$ such that

(1) the universal covering tree of $\mathbb{A}$ is $\left(q_{i_{1}}, q_{i_{2}}\right)$-biregular, where $m_{i_{1}, i_{2}}=\infty$, 


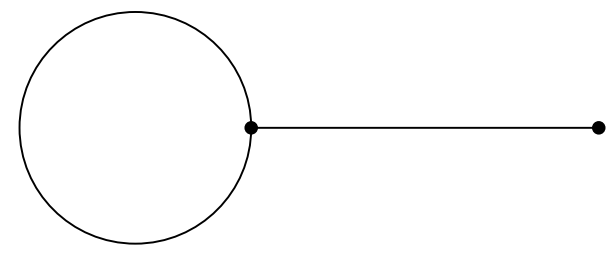

Figure 1: Rosenberg's graph for the case $q=3$

(2) the vertices of the graph $A$ may be 2-coloured, and

(3) the covolume of $\mathbb{A}$ equals $\frac{a}{b}$.

Suppose first that all $q_{i}$ are equal, and let $T_{q}$ be the $q$-regular tree.

If all $q_{i}=2$, we construct a graph of groups with universal covering tree $T_{2}$ and edge covolume $\frac{a}{1}=a$, for any integer $a \geq 1$. Let $A$ be the graph which is a ray of $a$ edges. The vertices of $A$ are then 2-colourable. Let $\mathbb{A}=(A, \mathcal{A})$ be the graph of groups over $A$ with each edge group trivial, the two vertex groups at either end of the ray both $\mathbb{Z} / 2 \mathbb{Z}$, and all other vertex groups trivial. Since the edge groups are trivial this graph of groups is faithful. Its universal covering tree is $T_{2}$ and it has covolume $a$.

If all $q_{i}=q$ are equal and $q \geq 3$, we require a graph of groups with universal covering tree $T_{q}$ and covolume $\frac{a}{b}$, where the prime divisors of $b$ are strictly less than $q$. This is exactly the set of covolumes of uniform lattices in $\operatorname{Aut}\left(T_{q}\right)$ (see Rosenberg [17, Proposition 9.1.2]). For $q \geq 4$, the vertices of each of the graphs in Rosenberg's proof are 2-colourable. In the case $q=3$, though, some of the graphs of groups are over the graph shown in Figure 1. The vertices of this graph cannot be 2-coloured.

A key idea of Rosenberg's proof, which is also used below to prove (1)(b), is that of finite-sheeted covers. In each case, the graph $A$, considered as a topological space, is not simply connected. The fundamental group $\pi_{1}(A)$ is then a free group on finitely many generators, so has subgroups of arbitrary finite index. Hence $A$ has a finitesheeted topological cover for any finite number of sheets. Thus, if a uniform lattice constructed over $A$ has covolume $\frac{a}{b}$, we may construct a uniform lattice with covolume a positive integer multiple of $\frac{a}{b}$, over a finite-sheeted cover of $A$.

In particular, the 2-sheeted cover of the graph in Figure 1 has 4 vertices, which may be 2-coloured. As explained in [17, Example 4, Chapter 4], the edge groups of this graph of groups have order a power of 2 , and this power may be chosen arbitrarily. Thus, by taking a 2 -sheeted cover and then doubling the order of the edge groups, we 
obtain a graph of groups over a 2-colourable graph with unchanged covolume. This completes the proof of (1)(a) in the case where all $q_{i}$ are equal.

Now suppose the $q_{i}$ are not all equal, and let $q_{i_{1}}=\max _{i}\left\{q_{i}\right\}$. By assumption, there is a $q_{i_{2}}$ with $m_{i_{1}, i_{2}}=\infty$. If $q_{i_{1}}=q_{i_{2}}$, then use the same graphs of groups as in the case where all $q_{i}$ are equal. Otherwise, to simplify notation, put $p=q_{i_{1}}$ and $q=q_{i_{2}}$, so that $p>q \geq 2$, and let $T_{p, q}$ be the $(p, q)$-biregular tree.

Rosenberg [17, Theorem 9.2.1] shows that the set of covolumes of uniform lattices in $\operatorname{Aut}\left(T_{p, q}\right)$ is the set of rationals $\frac{a}{b}$ such that the prime divisors of $b$ are strictly less than $p$. As with regular trees, for each such $\frac{a}{b}$, there is a faithful graph of finite groups, over a finite graph, with covolume $\frac{a}{b}$. Since each graph is covered by $T_{p, q}$, and $p \neq q$, the vertices of each graph are 2-colourable. This completes the proof of (1)(a).

3.1.2 Proof of (1)(b) By the proof of the Functor Theorem and Corollary 3.1, it suffices to prove Proposition 3.2 below.

Proposition 3.2 Let $T$ be a regular or biregular tree, other than the 2-regular tree. Let $v>0$ be the covolume of a uniform lattice in $\operatorname{Aut}(T)$. Then there is a countably infinite number of nonconjugate uniform lattices in $\operatorname{Aut}(T)$ which have covolume $v$.

Proof Since there are only countably many finite graphs of finite groups, there are at most countably many uniform lattices in $\operatorname{Aut}(T)$.

Assume that $T$ is the $m$-regular tree, for $m \geq 3$ (the proof for biregular trees is similar). Rosenberg [17, Proposition 9.1.2] constructs a faithful graph of finite groups $\mathbb{A}=(A, \mathcal{A})$ over a finite graph $A$ such that

(1) the universal covering tree of $\mathbb{A}$ is $T$,

(2) the fundamental group $\Gamma$ of $\mathbb{A}$ has covolume $v$,

(3) the vertices of the graph $A$ may be 2-coloured (in the case where $T$ is 3 -regular, use a $2-$ sheeted cover),

(4) the graph $A$ is not simply connected, and

(5) the orders of the edge and vertex groups $\mathcal{A}_{e}$ and $\mathcal{A}_{v}$ may be multiplied by powers of primes strictly less than $m$, and the new graph of groups is faithful.

Let $n$ be a positive integer whose prime divisors are strictly less than $m$. By (4), we may obtain a uniform lattice in $\operatorname{Aut}(T)$ of covolume $n v$. Applying (5), we may obtain a uniform lattice in $\operatorname{Aut}(T)$ of covolume $v / n$. By carrying out these two processes at the same time, we obtain a new lattice $\Gamma^{\prime}$ of covolume $v$, which is nonconjugate to the original lattice $\Gamma$, since the graph $\Gamma^{\prime} \backslash T$ is not the same as $A$. This may be done for countably many values of $n$. Hence we obtain countably many nonconjugate uniform lattices of covolume $v$. 


\subsection{Nonuniform covolumes}

3.2.1 Proof of (2)(a) Let $T$ be a regular or biregular tree, other than the 2-regular tree, and let $v>0$. By Corollary 3.1 and the proof of the Functor Theorem, it suffices to construct a nonuniform lattice $\Gamma$ in $\operatorname{Aut}(T)$ of covolume $v$, such that the vertices of the graph $\Gamma \backslash T$ may be 2-coloured.

Bass-Lubotzky showed that for $m \geq 3$ and every $v>0$ there is a nonuniform lattice $\Gamma$ acting on the $m$-regular tree $T_{m}$, such that the covolume of $\Gamma$ is $v$ (see Bass and Lubotzky [3, Theorem 4.3]). Moreover, the graph $\Gamma \backslash T_{m}$ is a ray, hence its vertices are 2-colourable. Rosenberg [17, Theorem 8.2.2] showed the analogous result for biregular trees, without the conclusion that the quotient graph is a ray. Since the vertices of any graph covered by a biregular tree $T_{m, n}$, with $m \neq n$, are 2-colourable, this completes the proof of (2)(a) of the Main Theorem.

3.2.2 Proof of (2)(b) Let $T$ be the $(m, n)$-biregular tree, with $m, n \geq 3$. Farb and Hruska [9] constructed commensurability invariants for nonuniform lattices acting on $T$. One such invariant is growth type.

As in [9, Section 3.2], let $f, g: \mathbb{N} \rightarrow \mathbb{N}$ be any two functions. We say that $f \preceq g$ if for some $\alpha, \beta \in \mathbb{N}$,

$$
f(k) \leq \alpha g(k+\beta)
$$

and we say that $f$ and $g$ are equivalent if $f \preceq g$ and $g \preceq f$.

Let $Y$ be a locally finite graph with basepoint $*$, and let $g: \mathbb{N} \rightarrow \mathbb{N}$ be such that $g(k)$ is the number of vertices in the combinatorial ball of radius $k$ centred at $*$. The growth type of $(Y, *)$ is defined to be the equivalence class of the function $g$. The growth type is independent of the choice of basepoint. If $\Gamma$ and $\Gamma^{\prime}$ are commensurable in $\operatorname{Aut}(T)$, then using finite covers one sees that the graphs $\Gamma \backslash T$ and $\Gamma^{\prime} \backslash T$ have the same growth type [9, Proposition 3.4].

The growth type of a lattice $\Gamma$ in $\operatorname{Aut}(X)$ may be similarly defined. The ball of radius 1 around a basepoint $*$ in $\Gamma \backslash X$ consists of all images of chambers which meet $*$, and by induction the ball of radius $k$ consists of all images of chambers which meet the ball of radius $k-1$. This growth type is also a commensurability invariant, by a similar proof to that for tree lattices.

Using growth type, for any $v>0$, there are uncountably many commensurability classes of nonuniform lattices with covolume $v$ in $\operatorname{Aut}(T)$ [9, Theorem 5.2]. These lattices are constructed using graphs of groups over particular trees. We now show that $F$ in the Functor Theorem, at least when applied to a tree, preserves growth type: 
Lemma 3.3 Let $Y$ be an (infinite) tree and let $F$ be as in the Functor Theorem. Then the growth type of $Y$ is the same as the growth type of the polyhedral complex $F(Y)$.

Proof Choose a basepoint $*$ of $Y$ and let $g(k)$ be the number of vertices in the combinatorial ball of radius $k$ centred at $*$. Let $G(k)$ be the number of vertices in the combinatorial ball of radius $k$ centred at the image of $*$ in $F(Y)$. We claim that $g$ is equivalent to $G$.

Let $l$ be the number of vertices of $P^{\prime}$, and for $j=1,2$ let $l_{j}$ be the number of vertices of the $i_{j}$-face of $P^{\prime}$. Without loss of generality we may assume that $l_{1} \geq l_{2}$ and that * has type $i_{1}$. Put $C=l_{1}-l_{2} \geq 0$. Since $Y$ is a tree, we then compute

$$
G(k)=\left(l-l_{j}\right) g(k)-l+2 l_{1}+C \sum_{m=1}^{k-1}(-1)^{m} g(m)
$$

where $j=1$ if $k$ is odd and $j=2$ if $k$ is even.

To show $G \preceq g$, by induction on $k$ we have

$$
\sum_{m=1}^{k-1}(-1)^{m} g(m) \leq g(k)
$$

Choose $\alpha$ so that $l-l_{j}+C \leq \alpha$ for $j=1,2$. Then choose $\beta$ so that $-l+2 l_{1} \leq \alpha \beta$. As $Y$ is an infinite tree, $\beta \leq g(k+\beta)-g(k)$ for all $k$. Hence

$$
G(k) \leq \alpha g(k)-l+2 l_{1} \leq \alpha g(k+\beta)
$$

This proves $G \preceq g$.

To show $g \preceq G$, by (3-1) we have

$$
g(k)=\frac{1}{l-l_{j}} G(k)+\frac{l-2 l_{1}}{l-l_{j}}-\frac{C}{l-l_{j}} \sum_{m=1}^{k-1}(-1)^{m} g(m)
$$

If $k$ is odd, then by induction

$$
-\sum_{m=1}^{k-1}(-1)^{m} g(m)=g(1)-g(2)+\cdots-g(k-1) \leq g(1)+0
$$

hence

$$
g(k) \leq \frac{1}{l-l_{1}} G(k)+\frac{l-2 l_{1}}{l-l_{1}}+\frac{C}{l-l_{1}} g(1)
$$

Algebraic 83 Geometric Topology, Volume 6 (2006) 
If $k$ is even then a similar inequality to (3-2) yields

$$
g(k) \leq \frac{1}{l-l_{2}} G(k)+\frac{l-2 l_{1}}{l-l_{2}}+\frac{C}{l-l_{2}}[g(1)+g(k)]
$$

Since $C=l_{1}-l_{2}$, we have $1-\frac{C}{l-l_{2}}=\frac{l-l_{1}}{l-l_{2}}>0$. On rearranging (3-4), we thus obtain the inequality (3-3) for $k$ even as well. Using (3-3), we may then choose suitable constants $\alpha$ and $\beta$, and conclude that $g \preceq G$.

By the proof of the Functor Theorem, it follows that for any $v>0$ there are uncountably many commensurability classes of nonuniform lattices in $\operatorname{Aut}(X)$ of covolume $v$. This completes the proof of (2)(b).

\subsection{Towers}

We construct towers in $\operatorname{Aut}(X)$ using full subcomplexes of subgroups, as explained in Section 1.4.1. Let $T$ be a biregular tree, with at least one valence greater than 2. By the proof of the Functor Theorem, it suffices to find a tower of tree lattices in $\operatorname{Aut}(T)$, constructed as a sequence of full subgraphs of subgroups, over a graph $A$ whose vertices may be 2 -coloured.

3.3.1 Proof of (3)(a) Let $m$ and $q$ be integers $\geq 2$. Bass and Kulkarni [2, Proposition 7.15], give an example of a tower of uniform tree lattices acting on the $(m+1, q)-$ biregular tree. The vertices of the graph $A$ in this example may be 2-coloured.

3.3.2 Proof of (3)(b) An indexing on a graph $A$ is a map $I$ from the edges of $A$ to the positive integers. A graph of groups $\mathbb{A}=(A, \mathcal{A})$ over $A$ induces the indexing $I(e)=\left[\mathcal{A}_{i(e)}: \alpha_{e}\left(\mathcal{A}_{e}\right)\right]$. We say that an indexed graph $(A, I)$ admits a tower of lattices if there exists a sequence of full subgraphs of subgroups over $A$ which induces a tower of tree lattices, such that each graph of groups in the sequence induces the indexing $I$.

Carbone and Rosenberg [8, Theorem 5.3] give a sufficient condition for $(A, I)$ to admit a tower of nonuniform tree lattices. It is easy to construct examples of $(A, I)$ which satisfy this condition, so that the tower of tree lattices acts on the regular or biregular tree, and the vertices of $A$ are 2-colourable.

3.3.3 Proof of (3)(c) We apply [2, Example 7.13]. The graph $A$ in this construction is a single edge, so its vertices are 2-colourable. The universal covering tree is the $(m p, q)$-biregular tree, where $m, p$ and $q$ are positive integers $\geq 2$. Putting $q_{i}=m p$ which is composite, there exists a tower of uniform lattices in $\operatorname{Aut}(X)$ with quotient a single chamber.

Algebraic 83 Geometric Topology, Volume 6 (2006) 


\section{References}

[1] H Bass, Covering theory for graphs of groups, J. Pure Appl. Algebra 89 (1993) 3-47 MR1239551

[2] H Bass, R Kulkarni, Uniform tree lattices, J. Amer. Math. Soc. 3 (1990) 843-902 MR1065928

[3] H Bass, A Lubotzky, Tree lattices, Progress in Mathematics 176, Birkhäuser, Boston (2001) MR1794898

[4] A Borel, On the set of discrete subgroups of bounded covolume in a semisimple group, Proc. Indian Acad. Sci. Math. Sci. 97 (1987) 45-52 (1988) MR983603

[5] M Bourdon, Immeubles hyperboliques, dimension conforme et rigidité de Mostow, Geom. Funct. Anal. 7 (1997) 245-268 MR1445387

[6] M R Bridson, A Haefliger, Metric spaces of non-positive curvature, Grundlehren series 319, Springer, Berlin (1999) MR1744486

[7] M Burger, S Mozes, Lattices in product of trees, Inst. Hautes Études Sci. Publ. Math. (2000) 151-194 (2001) MR1839489

[8] L Carbone, G Rosenberg, Infinite towers of tree lattices, Math. Res. Lett. 8 (2001) 469-477 MR1849263

[9] B Farb, G C Hruska, Commensurability invariants for nonuniform tree lattices, Israel J. Math. 152 (2006) 125-142 MR2214456

[10] D Gaboriau, F Paulin, Sur les immeubles hyperboliques, Geom. Dedicata 88 (2001) 153-197 MR1877215

[11] Y Glasner, A two-dimensional version of the Goldschmidt-Sims conjecture, J. Algebra 269 (2003) 381-401 MR2015283

[12] D M Goldschmidt, Automorphisms of trivalent graphs, Ann. of Math. (2) 111 (1980) 377-406 MR569075

[13] F Haglund, F Paulin, Constructions arborescentes d'immeubles, Math. Ann. 325 (2003) 137-164 MR1957268

[14] T Januszkiewicz, J Świątkowski, Hyperbolic Coxeter groups of large dimension, Comment. Math. Helv. 78 (2003) 555-583 MR1998394

[15] A Lubotzky, Tree-lattices and lattices in Lie groups, from: "Combinatorial and geometric group theory (Edinburgh, 1993)", London Math. Soc. Lecture Note Ser. 204, Cambridge Univ. Press (1995) 217-232 MR1320284

[16] L Potyagailo, E Vinberg, On right-angled reflection groups in hyperbolic spaces, Comment. Math. Helv. 80 (2005) 63-73 MR2130566

[17] G E Rosenberg, Towers and Covolumes of Tree Lattices, PhD thesis, Columbia University (2001) 
[18] J-P Serre, Cohomologie des groupes discrets, from: "Prospects in mathematics (Proc. Sympos. Princeton 1970)", Ann. of Math. Studies 70, Princeton Univ. Press (1971) 77-169 MR0385006

[19] J-P Serre, Trees, Springer, Berlin (1980) MR607504

[20] A Thomas, Covolumes of uniform lattices acting on polyhedral complexes, Bull. London Math. Soc. to appear

[21] ̇̀ B Vinberg, O V Shvartsman, Discrete groups of motions of spaces of constant curvature, from: "Geometry II: Spaces of Constant Curvature", Encyclopaedia Math. Sci. 29, Springer, Berlin (1993) 139-248 MR1254933

Department of Mathematics, University of Chicago

5734 South University Ave, Chicago IL 60637, USA

athomas@math . uchicago . edu

Received: 3 March 2006 Revised: 29 June 2006 